This is the accepted manuscript of the article, which has been published in CMBEBIH 2019 : Proceedings of the International Conference on Medical and Biological Engineering. 2019, 7-11.

CMBEBIH2019, 015, v2 (final): 'Electrical Stimulation of Eye Blink in Individuals with...

\title{
Electrical Stimulation of Eye Blink in Individuals with Dry Eye Symptoms Caused by Chronic Unilateral Facial Palsy
}

\author{
J. Lylykangas ${ }^{1}$, M. Ilves ${ }^{1}$, H. Venesvirta ${ }^{3}$, V. Rantanen ${ }^{2,3}$, E. Mäkelä ${ }^{3,4}$, \\ A. Vehkaoja ${ }^{2}$, J. Verho $^{2}$, J. Lekkala ${ }^{2}$, M. Rautiainen ${ }^{3}$, and V. Surakka ${ }^{1}$ \\ ${ }^{1}$ Faculty of Information Technology and Communication Sciences, \\ Tampere University, Tampere, Finland \\ ${ }^{2}$ BioMediTech Institute and Faculty of Medicine and Health Technology, \\ Tampere University, Tampere, Finland \\ ${ }^{3}$ Faculty of Medicine and Health Technology (Otorhinolaryngology), \\ Tampere University, Tampere, Finland \\ ${ }^{4}$ Department of Clinical Neurophysiology, Medical Imaging Centre, \\ Pirkanmaa Hospital District, Tampere, Finland
}

\begin{abstract}
The aim was to validate the functionality and subjective experiences of timer-triggered electrical blink stimulation with participants $(\mathrm{N}=6)$ suffering from dry eye symptoms caused by chronic unilateral facial palsy. In a stimulation condition, the muscles responsible for eye blinking were stimulated at fixed intervals while watching a video for about 120 minutes. In a control condition, the participants watched a video without stimulation. The participants rated their dry eye symptoms with a questionnaire before and after the both conditions. They also rated the levels of felt pain, discomfort and naturalness of the stimulated movement. Additionally, the magnitude of the stimulated eye blinks over time was evaluated. The results showed that the magnitude of the stimulated eye blink did not decrease significantly during the watching task. The stimulation was rated as painless, slightly uncomfortable, and fairly natural. The experienced eye dryness decreased significantly in the stimulation condition. Most participants got used to the stimulation, or even forgot it during the task. The findings are promising in respect to the use of timer-triggered blink stimulation.
\end{abstract}

KEYWORDS: Facial palsy; Eye blink; Dry eye disease; Dry eye symptoms; Electrical stimulation.

\section{Introduction}

Facial nerve palsy affects the function of facial muscles, including the muscles responsible for eye blinking. The paresis is most typically unilateral affecting one side of the face while the intact side functions normally. Eye blinking is crucial for clear vision as well as protecting, lubricating, and cleaning the eye. The impaired ability to blink often leads to dry eye disease, which causes corneal discomfort and blurred vision [3]. Typical treatment for the symptoms is the use of eye drops to lubricate the eye surface. In 
more severe cases of facial palsy, surgical operations are required to avoid corneal damage. The main deficits of surgery include altered facial appearance (e.g., weight attached to the upper eyelid or eyelids sewed together) and a risk of complications.

In recent years, a method called facial pacing has been studied $[7,10]$. The idea is to detect eye blink-related signals from the healthy side of the face and use this measurement to trigger a concurrent eye blink on the paretic side. Frigerio et al. [7] found that transcutaneous electric stimulation of the facial nerve branches of orbicularis oculi evoked eye blink in about $50 \%$ of the patients with acute facial palsy. However, the reliable detection of eye blinks may be challenging even in controlled laboratory settings [e.g., 5]. For this reason, simpler methods are worth considering.

In our previous study [9] we introduced a timer-triggered, constant-interval blink stimulator as an alternative for a blink-triggered pacing system. Our results with healthy participants showed that the pre-timed blink stimulation method was functional, and it was rated positively after a relatively long visual task.

The current clinical trial was carried out to validate the functionality of the method stated above with participants who suffered from dry eye symptoms caused by chronic unilateral facial palsy. The aim was to study subjective experiences of the stimulation and its effects on dry eye symptoms. Additionally, we observed the magnitude of the stimulated eye blinks regarding the possible changes in long-term use, for example, due to muscle fatigue.

\section{Methods}

\subsection{Participants}

Six volunteers (5 females, 1 male) with chronic peripheral facial nerve palsy were enrolled to the study from the participant pool of our previous experiments. Their age ranged between 25 and 53 years (mean $=44.2, \mathrm{SD}=10.5$ ). The etiology of the palsy included Bell's palsy $(\mathrm{n}=4)$, Ramsey-Hunt syndrome $(\mathrm{n}=1)$, and suspected Lyme disease $(n=1)$. They all had earlier experience of transcutaneous electrical stimulation of facial muscles. The inclusion criteria included successful orbicularis oculi activation induced by electrical stimulation and self-reported complications on eye dryness. The participants signed an informed consent form prior to the experiment. The study was accepted by the ethical committee of Pirkanmaa Hospital District (R15067). Participation was compensated with cafeteria tickets.

\subsection{Assessment of the palsy and ocular symptoms}

Severity of the palsy was individually assessed with the Sunnybrook facial grading system (SFGS) [12]. The mean SFGS scores varied between 25 and 76 (mean $=40.3$, SD = 16.3). Participants' Ocular Surface Disease Index (OSDI; (C) Allergan Inc., Irvine, California, USA) [13] score was between 10.4 and $66.7($ mean $=46.6, \mathrm{SD}=19.5)$. The scores indicated moderate dry eye disease, except for the participant with the lowest score, whose symptoms were primality experienced during outdoor sports. 


\subsection{Equipment and stimulation parameters}

The stimulation device included four channels for functional electrical stimulation. The device communicated wirelessly with a PC, which was also used to adjust the stimulation parameters (for a detailed description of the stimulation device, see [11]). The stimulation waveform used in this study was a biphasic square wave with symmetric positive and negative phases (equal width, equal amplitude) using phase duration of $0.4 \mathrm{~ms}$ and $250 \mathrm{~Hz}$ pulse repetition frequency. Pulse train duration was $200 \mathrm{~ms}$ with ramping time of $80 \mathrm{~ms}$. The parameters were selected based on earlier research [e.g., 6, 7, 9, 9]. The commercial, disposable stimulation electrodes made from carbonized rubber were manually trimmed approximately to a size of $1.9 \mathrm{~cm}^{2}$. Two electrodes were attached to the skin above the orbicularis oculi muscle. One electrode was positioned $0.5 \mathrm{~cm}$ lateral to the orbital rim and the other about $0.5 \mathrm{~cm}$ posterior to the first one. The participant's face was recorded with a digital video camera at 50 frames per second for offline video analysis.

\subsection{Procedure}

The experiment was carried out during two separate days with a maximum of one day in between. One visit with and another without blink stimulation (stimulation and control conditions, respectively) were counterbalanced between the participants so that half started with stimulation and the other half with the control condition.

In the beginning of the first visit, the aim of the study was explained and the participant signed an informed consent and a consent for the use of video material. Then the SFGS was obtained, and the participant filled out the OSDI questionnaire. In addition, the possible immediate effects of the stimulation and/or the video watching on subjective eye dryness symptoms were rated with a custom dry eye questionnaire (DEQ), which was made based on Begley et al. [1, 2]. The DEQ assessed nine typical dry eye symptoms (watering, itching, stinging, foreign body sensation, pain, dryness, sensitivity to light, changes in eye sight, and general discomfort) by five-point scales varying from 1 (not at all) to 5 (a lot). A vision test for both eyes was performed using a logarithm of the minimum angle of resolution (logMAR) chart.

At first, the muscle of the thenar eminence of a participant's left hand was stimulated in order to familiarize the participant to the stimulation. The familiarization continued by stimulating the orbicularis oculi muscle on the healthy side. The stimulation started from $1.0 \mathrm{~mA}$ and it was increased in $0.5 \mathrm{~mA}$ steps. The stimulus was repeated five times at each amplitude level. The participant's permission to proceed was requested at each level until a full eye closure was evoked. The threshold for the eye closure (i.e., a complete blink) was visually estimated real time by two experimenters.

In the stimulation condition, the rating scales were explained and practiced. Both the pain and the discomfort rating scales ranged from 0 (not at all painful / discomfortable) to 10 (extremely painful / discomfortable). The movement naturalness scale was an eleven-point bipolar rating scale varying from -5 (unnatural) to +5 (natural), with 0 representing the neutral point (neither unnatural nor natural) of the scale. The stimulation was continued with the paretic side by following a similar procedure in order to 
find suitable eye closure threshold. Stimulus amplitude for a full eye closure was confirmed onsite in an offline frame-by-frame video analysis. Using this personal amplitude level, the stimulation was repeated five times with a five-second interstimulus interval, and the participant gave the pain, discomfort, and naturalness ratings regarding the stimulation. Then, the participant watched a freely chosen video for approximately 120 minutes from a 50 -inch LCD television at a 1.85 -meter viewing distance. During the watching, the orbicularis oculi muscle was stimulated at five-second intervals (see [9] for the rationale of the selected stimulus rate). After the watching task, the participant gave again the pain, discomfort, and the naturalness ratings.

In the control condition, familiarization with healthy side stimulation was followed by paretic side orbicularis oculi stimulation until the full eye closure threshold was reached. Then the electrodes were removed, and the participant proceeded to the video watching task without the stimulation.

In the end of both conditions, the participant again filled out the DEQ and repeated the vision test. Corneal health status was examined with a fluorescein eye stain test, except for one participant who experienced the test too uncomfortable. Before leaving the stimulation condition, the participant was interviewed about the following topics: was the stimulation noticeable, did it disturb watching the video, did the feeling about the stimulation change over the time, did the stimulation have any effect on the experienced dry eye symptoms, and was the stimulated blink rate appropriate. The experiment took approximately 3.5 hours per visit.

The procedures of the stimulation and control conditions were similarly arranged in the second visit, excluding the consent forms, SFGS, and OSDI, which were collected only in the first visit. Additionally, control conditions performed in the second visit excluded the stimulus familiarization.

\subsection{Data analysis}

The thresholds for eyelid movement and complete eye closure on the healthy and paretic side of the face were obtained in offline video analysis by one experimenter. Electrical currents for the thresholds were analyzed with non-parametric Wilcoxon signed rank tests.

One-way repeated measures analysis of variance (ANOVA) with blink magnitude as a factor was used to test whether the blink magnitude changed during the watching task. The magnitude of the blinks was classified in offline video analysis by one experimenter. The magnitude was determined from five stimulated blinks in every 10 minutes with no concurrent eyelid movement observed on the healthy side. The classification from the largest to the lowest was $5=$ complete blink; $4=$ nearly complete blink; $3=$ eyelid covers the pupil; $2=$ eyelid partially covers the pupil; $1=$ twitch.

Wilcoxon signed rank tests were used to analyze subjective ratings on stimulation, DEQ, and the visual acuity test. For the DEQ and visual acuity test, the comparisons between before and after the video watching task were performed separately for the two conditions. 


\section{$3 \quad$ Results}

Stimulation threshold to produce an eyelid movement on the healthy and paretic side varied between 2.0-3.5 mA (mean $=2.5, \mathrm{SD}=0.5)$ and 2.5-3.0 $\mathrm{mA}($ mean $=2.8, \mathrm{SD}$ $=0.3$ ), respectively. Wilcoxon signed rank test did not show a statistically significant difference between these stimulation thresholds.

Stimulation thresholds for a complete eye blink on the healthy and paretic side varied between 2.5-5.0 $\mathrm{mA}($ mean $=3.4, \mathrm{SD}=0.9)$ and 4.0-7.0 $\mathrm{mA}($ mean $=5.3, \mathrm{SD}=1.0)$, respectively. Wilcoxon signed rank test showed that the threshold was significantly higher for the paretic than healthy side $\mathrm{Z}=-2.21, \mathrm{p}<.05$.

Figure 1 shows the mean eye blink magnitudes of the stimulated blinks as a function of time. One-way repeated measures ANOVA showed no significant change in the blink magnitude during the watching task.

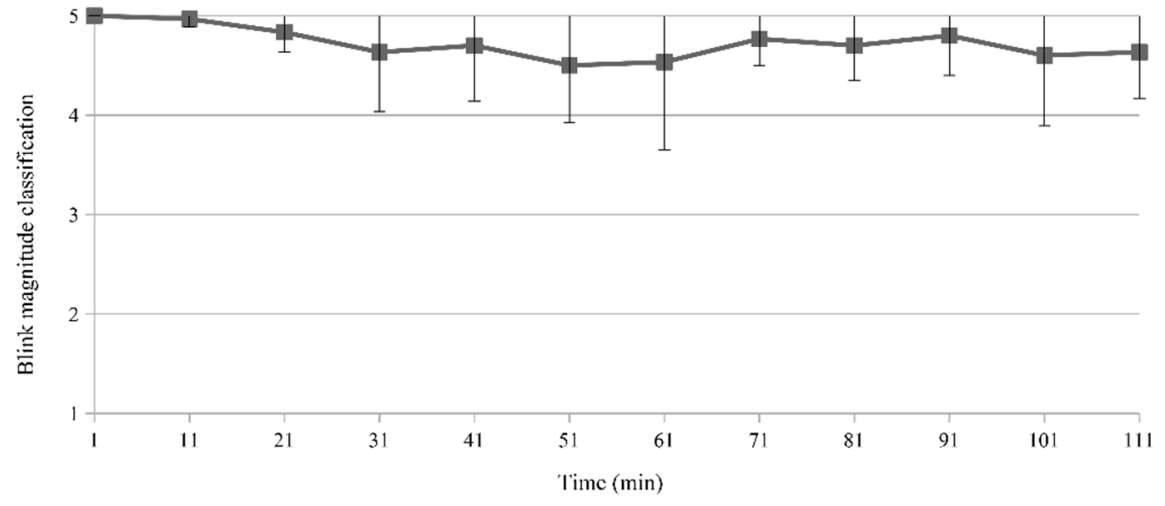

Fig. 1. Classified mean eye blink magnitudes of the stimulated blinks as a function of time. Error bars represent SDs.

Table 1 shows the results from the DEQ. Wilcoxon signed rank test showed that the sensation of dryness was significantly lower after the stimulation than before it, $\mathrm{Z}=$ $2.12, \mathrm{p}<.05$. Other pairwise comparisons were not statistically significant. 
Table 1. The mean ratings from the DEQ scales with SDs in parenthesis. The grey background indicates the statistically significant difference.

\begin{tabular}{|c|c|c|c|c|}
\hline & \multicolumn{2}{|c|}{ Stimulation condition } & \multicolumn{2}{|c|}{ Control condition } \\
\hline & Before video & After video & Before video & After video \\
\hline Watering & $2.2(1.2)$ & $2.0(0.9)$ & $2.2(1.5)$ & $2.0(1.5)$ \\
\hline Itching & $2.2(1.0)$ & $1.7(1.2)$ & $1.8(1.3)$ & $2.0(1.3)$ \\
\hline Stinging & $1.5(0.8)$ & $2.0(1.3)$ & $1.7(1.0)$ & $2.5(1.0)$ \\
\hline Foreign body sensation & $3.2(1.2)$ & $1.8(1.6)$ & $2.0(1.1)$ & $3.0(1.5)$ \\
\hline Pain & $2.2(1.2)$ & $1.8(1.3)$ & $2.2(1.3)$ & $2.7(1.4)$ \\
\hline Dryness & $3.5(1.2)$ & $2.2(1.3)$ & $2.7(1.6)$ & $3.5(2.0)$ \\
\hline Sensitivity to light & $2.2(1.6)$ & $2.0(1.3)$ & $1.8(1.3)$ & $2.0(1.5)$ \\
\hline Changes in eye sight & $2.5(1.2)$ & $2.8(1.3)$ & $2.2(1.8)$ & $2.2(1.2)$ \\
\hline Discomfort & $3.7(0.8)$ & $2.7(1.6)$ & $2.3(1.2)$ & $3.0(1.5)$ \\
\hline
\end{tabular}

Figure 2 shows the ratings about the stimulation pain and discomfort, and Figure 3 shows the ratings for the movement naturalness before and after the video watching task. Wilcoxon signed rank tests showed no statistically significant differences for the ratings.

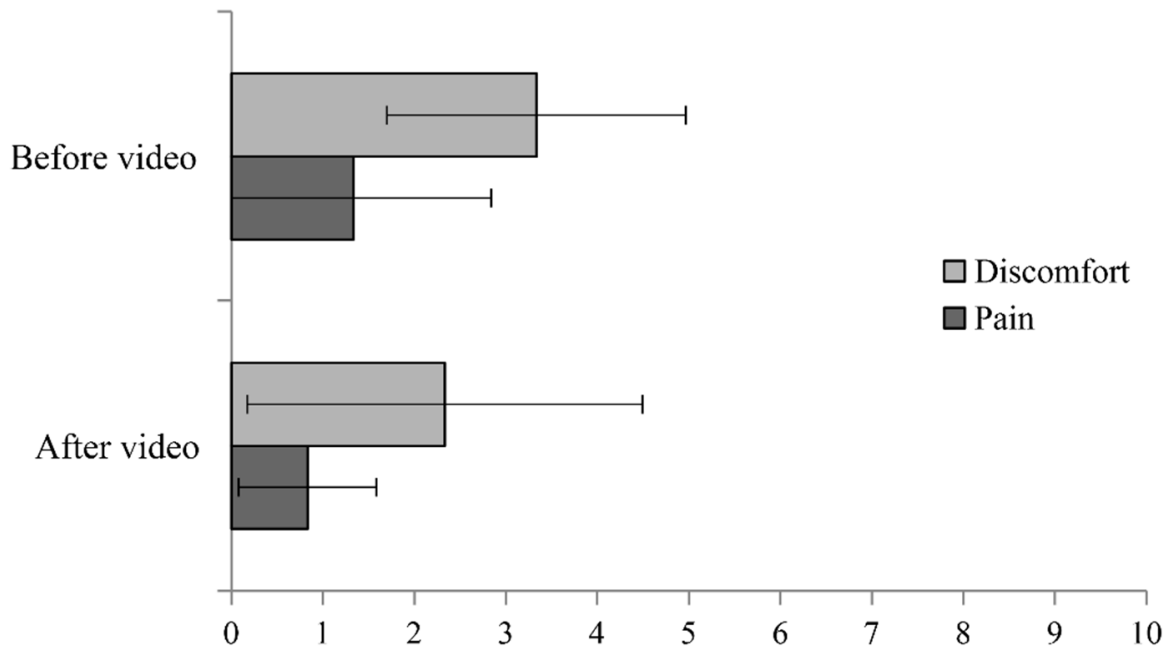

Fig. 2. The mean discomfort and pain ratings for blink stimulation before and after the watching task. Error bars represent SDs. 
Before video

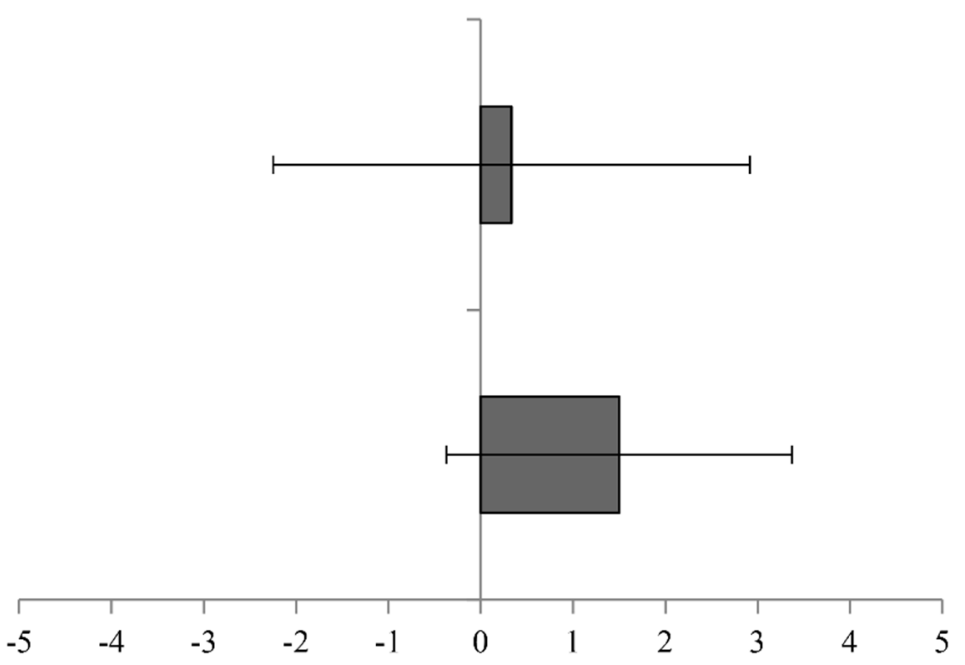

Fig. 3. The mean movement naturalness ratings for blink stimulation before and after the watching task. Error bars represent SDs.

Wilcoxon signed rank tests did not reveal significant differences of visual acuity in stimulation and control conditions. The fluorescein eye stain tests showed that the corneal status remained the same in the stimulation and control conditions.

In the post-experimental interview, four of the six participants reported that they forgot the stimulation during the watching task or got used to it. Two said that the stimulation reduced their dry eye symptoms. Five participants requested that the interval of the stimulated blinks should be modifiable. Three were interested to try out a portable blink stimulator in everyday use if available. Two participants mentioned that the stimulation had a positive effect on the eye symptoms, and even further, one who suffered from prominent watering symptom considered having less need to wipe the tears below the paretic-side eye during the stimulation.

\section{Discussion}

The results showed that timed eye blink stimulation can be useful and acceptable for individuals with dry eye symptoms caused by chronic facial palsy. The stimulation method used in the study is simpler than facial pacing in earlier efforts [7, 10]. Pacing can be useful if synchrony of eye blinks between the eyes is required, but in many cases a simpler and more cost-effective method may be favorable.

Although complete eye blink in the paretic side required significantly higher stimulus amplitude compared to the healthy side, the stimulation was not felt as painful. The ratings of pain and discomfort were even slightly lower after the two-hour video watching task than before it, and the movement naturalness was rated higher after the watching. Further, most participants reported that they got used to stimulation or even forgot 
it while watching the video. These subjective experiences of adjustment to the stimulation are in line with those obtained from intact participants in our previous study [9].

The five-second interstimulus interval together with other stimulus parameters seemed to provide sufficient recovery time for the muscle fibers. The magnitude of the stimulated blinks did not decrease significantly during the watching task. This indicates that the stimulation did not cause noticeable muscle fatigue. This is an interesting finding compared to earlier studies, which state that high stimulation frequency may cause faster fatigue of limb muscles [4]. The relatively high $250 \mathrm{~Hz}$ frequency used for stimulating the orbicularis oculi in the current experiment did not show this kind of tendency. Thus, one of the future research topics would include systematic investigation on how different frequencies and other stimulus parameters affect to the fatigue of different facial muscles.

The subjective ratings indicated that the stimulated eye blink may reduce the dry eye symptoms caused by the palsy. The experienced dryness was significantly lower after the stimulation than before it, and additionally, itching, foreign body sensation, pain, light sensitivity, and overall discomfort symptoms had a tendency to decrease during the stimulation, whereas the tendency was to increase during the control condition.

Our next steps include usability and user experience studies with a mobile eye blink stimulator with enhanced functionality, including adjustable blink rate, in everyday use.

\section{Acknowledgments}

This study was funded by the Academy of Finland: decision numbers 278529, 276567, and 278312.

\section{Declarations of interest}

The authors declare that they have no competing interests.

\section{$7 \quad$ References}

1. Begley, C. G., Caffery, B., Chalmers, R. L., \& Mitchell, G. L. (2002). Use of the dry eye questionnaire to measure symptoms of ocular irritation in patients with aqueous tear deficient dry eye. Cornea, 21(7), 664-670.

2. Begley, C. G., Chalmers, R. L., Mitchell, G. L., Nichols, K. K., Caffery, B., Simpson, T., ,.. \& Davis, L. (2001). Characterization of ocular surface symptoms from optometric practices in North America. Cornea, 20(6), 610-618.

3. Craig, J. P., Nichols, K. K., Akpek, E. K., Caffery, B., Dua, H. S., Joo, C. K., ... \& Stapleton, F. (2017). TFOS DEWS II definition and classification report. The ocular surface, 15(3), 276-283.

4. Doucet, B. M., Lam, A., \& Griffin, L. (2012). Neuromuscular electrical stimulation for skeletal muscle function. The Yale journal of biology and medicine, 85(2), 201-215. 
5. Frigerio, A., Brenna, S., \& Cavallari, P. (2013). Surface electromyography recording of spontaneous eyeblinks: applications in neuroprosthetics. Otolaryngology--Head and Neck Surgery, 148(2), 209-214.

6. Frigerio, A., \& Cavallari, P. (2012). A closed-loop stimulation system supplemented with motoneurone dynamic sensitivity replicates natural eye blinks. Otolaryngology--Head and Neck Surgery, 146(2), 230-233.

7. Frigerio, A., Heaton, J. T., Cavallari, P., Knox, C., Hohman, M. H., \& Hadlock, T. A. (2015). Electrical stimulation of eye blink in individuals with acute facial palsy: progress toward a bionic blink. Plastic and reconstructive surgery, 136(4), 515e-523e.

8. Ilves, M., Lylykangas, J., Rantanen, V., Mäkelä, E., Vehkaoja, A., Verho, J., .. \& \& Surakka, V. (2019). Facial muscle activations by functional electrical stimulation. Biomedical Signal Processing and Control Journal, 48, 248-254.

9. Lylykangas, J., Ilves, M., Venesvirta, H., Rantanen, V., Mäkelä, E., Vehkaoja, A., ... \& Surakka, V. (2017). Artificial eye blink pacemaker-A first investigation into the blink production using constant-interval electrical stimulation. In EMBEC \& NBC 2017 (pp. 522-525). Springer, Singapore.

10. McDonnall, D., Guillory, K. S., \& Gossman, M. D. (2009). Restoration of blink in facial paralysis patients using FES. In Neural Engineering, 2009. NER'09. 4th International IEEE/EMBS Conference on (pp. 76-79). IEEE.

11. Rantanen, V., Vehkaoja, A., Verho, J., Veselý, P., Lylykangas, J., Ilves, M., ... \& Lekkala, J. (2016). Prosthetic pacing device for unilateral facial paralysis. In XIV Mediterranean Conference on Medical and Biological Engineering and Computing 2016 (pp. 653-658). Springer, Cham.

12. Ross, B. G., Fradet, G., \& Nedzelski, J. M. (1996). Development of a sensitive clinical facial grading system. Otolaryngology—Head and Neck Surgery, 114(3), 380-386.

13. Schiffman, R. M., Christianson, M. D., Jacobsen, G., Hirsch, J. D., \& Reis, B. L. (2000). Reliability and validity of the ocular surface disease index. Archives of ophthalmology, 118(5), 615-621. 